\title{
Gas flow-switching arc interaction in HV synchronous gas blast interrupters
}

\author{
Natalia Kurakina*, Vladimir Frolov and Eugeny Tonkonogov \\ Peter the Great St. Petersburg Polytechnic University, St. Petersburg, Russian Federation
}

\begin{abstract}
The paper is devoted to the solution of short-circuit switching problem in distribution networks by application of synchronous HV gas-blast circuit breakers (SCB). The reduction of the arcing energy by reducing the arcing time can extend the SCB expected service life by increasing its electrical life, minimizing the thermal requirements of the interrupting chamber. The paper studies the gas flow arrangement in the switching gap of the double-flow model SCB. Well-balanced and stable gas flow removes surplus energy from the switching arc. Three types of nozzle geometry are investigated. A numerical simulation with a grid reconstruction is used. A gas flow energy accumulation into the nozzle throats is observed at $t=0.8-0.9 \mathrm{~ms}$ after the contacts opening. We evaluate the bottom time limit of the active interactions between the gas flow and the arc column before current zero under the synchronous commutation. A zone of stagnation depends on the nozzle shape and size and determines the level of the thermal-dielectric interruption ability.
\end{abstract}

\section{Introduction}

The modern trends of increasing the rated voltage and short circuit breaking current per a break [1-2] with applying of alternative for SF6 medium [3-4] become an urgent problem for developers [5] and require new approaches for its solving.

An idea of synchronous commutation as the effective method to enhance the interruption ability of SCB is well known in scientific community [6-7]. A common principle of SCB was described in [7]. A synchronous pulse in periodically generated just before alternating current zero. Only after the tripping signal, the driving circuit for the breaking contact is excited by the synchronous pulse. The breaking contact is moved by the special drives. Controlled switching is realized by the arc contact SCB separation at the definite instant before the moment of current zero. The effective interactions between a switching arc and a gas flow removes the surplus energy from a gap between electrical contacts of the SCB. To provide it the gas flow should be wellbalanced and stable around current zero. In this case, the arcing time is significantly reduced, because the energy release during the arcing greatly decreases. The synchronous opening of fault currents can possibly increase the SCB interrupting capacity. However, there are problems with high proportion failures and insufficient response time attributed to the drives [8-10]. Furthermore, the contacts opening and gas introduction times under the synchronous commutation are controversial issues (see below).

The relationship between the rate of the rise of recovery voltage $d U / d t$ and the rate of the current fail before current zero $d I / d t$ usually are written as $d U / d t \sim(p)^{n}(d I / d t)^{-m}$, where $1<\mathrm{n}<1.6, \quad 1.5<\mathrm{m}<3 \quad$ are empirical constants. The determination of these empirical constants in adaptive arc model components and qualitative analysis of the processes in the vicinity of current zero will permit to increase the prediction level of clear/fail boundary. At the same time, a nozzle shape has a significant contribution to the interruption ability. Figure 1 presents a logarithmic relationship of parameter $R V^{*}=(d U / d t) / p_{c r}$ versus $d I / d t$ for air model SCB, where $p_{c r}=p / 2$. The data were received from experiments of different authors (1-2) [7], (3) [11], (4) [12], (5) [13] with various values of inlet pressure $p=27.46,14.71$, 5.85, 30, 37.5 bar and nozzle diameters $d_{N}=40,40,45$, $31.8,12.5 \mathrm{~mm}$ accordingly.

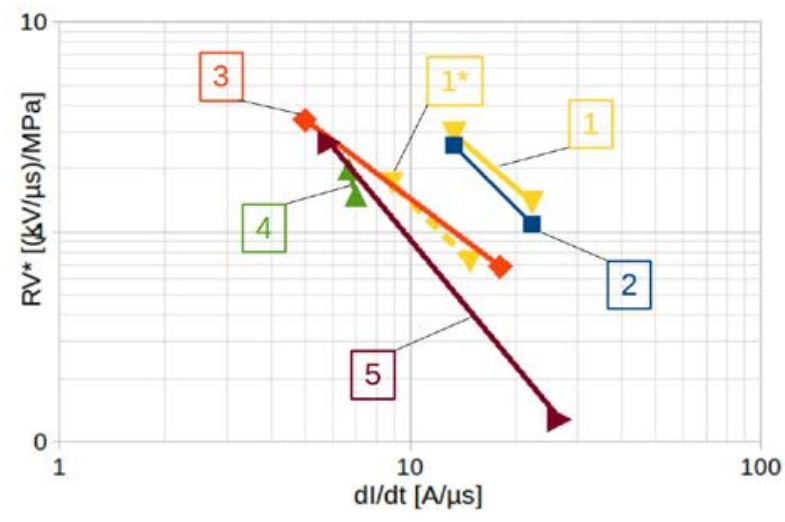

Fig. 1. Normalized parameter $R V^{*}$ as a function of $d I / d t$; (1-2) [7] - the double flow model SCB; (3) [11], (4) [12], (5) [13] the mono-flow model SCB.

\footnotetext{
* Corresponding author: nkuriee@gmail.com
} 
The analysis of the curves in fig. 1 shows the general trend of interaction ability for air blast circuit interrupters [14]. Yellow (1) and blue (2) curves were plotted for double flow model SCB. Their interrupting ability is by a factor of 1.5 higher than that of mono-flow devices, see curves (3), (4), and (5). Yellow dashed line $\left(1^{*}\right)$ denotes the "actual" location of the curve (1) after dividing by the over-coefficient 1.5 .

It the research [6] mono- and double-flow model SCB systems were compared (fig. 2).

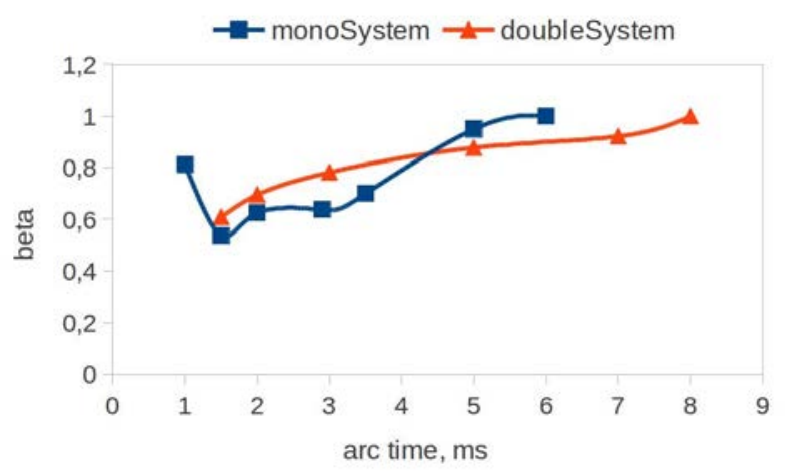

Fig. 2. Arcing time before current zero as a function of normalized parameter $\beta$.

The experimental curves reproduce in fig. 2, where a normalized parameter $\beta$ is the ratio of the limit extinguishing pressure under the existing experimental conditions with the synchronous commutation and limit extinguishing pressure for a common switching model. The limit extinguishing pressure for the model SCB was defined by the contacts opening in different phases before current zero. The results (fig.2) demonstrated an increase of the interruption ability for the model SCB with the contacts opening at the period of $1.5-3 \mathrm{~ms}$ before current zero. At $t<1.5 \mathrm{~ms}$ it was observed a decrease of the arc quenching performance. However, the arcing time in the study [7] was about 1.5-2 ms. This means a successful commutation took place before than $1.5 \mathrm{~ms}$. One of the possible reason of such inconsistency could depend upon a site of arc quenching (either into the nozzle throat or periphery) and the complicated arcgas correlations in the switching gap [15].

Therefore, well-balanced and stable gas flow across the nozzle throat contributes to the more effective arcgas interactions, reduces the surplus energy from the plasma residual column, provides the enhanced deionization between the electrical contacts, and avoids the negative effects, which influence of the interruption ability of the model SCB. Such negative factors include, for example, thermal clogging phenomena of the nozzle, an ablation of material from the contacts [16] due to unsteady gas-dynamics characteristics of the flow, a formation of high-temperature stagnation zones [17], and a thermal-dielectric breakdown after current zero.

The main interest of our study was to observe the beginning time of the effective arc-gas correlations under the synchronous contacts opening before current zero to increase the interruption ability of the SCB.

\section{Methods}

Numerical analysis has been performed for a model double-flow model SCB presented in fig. 3. The investigated device consists of a double nozzle system with one fixed and one moving parts. The gas of high pressure (dry air) introduces to the chamber via inlet when contacts opening start.

It is very important to choose a geometrically optimum arrangement of the nozzle in the system, taking into attention a minimum gas consumption with the efficient working pressure for the rated conditions of voltage recovery, necessary for arc quenching. The gas flow should be aerodynamically clean to provide the best conditions of coaxial stabilization of the arc column [18]. Therefore, in the calculation model in fig.3 following parameters for the nozzle shape are applied (as a first approximation): a nozzle critical section diameter is $d_{N}=40 \mathrm{~mm}$, a convergent nozzle throat (subsonic part) is $1.5 d_{N}$, its bending radius is not less than $d_{N}$, a divergent nozzle side angle (supersonic part) is less than 10 degree. Systems with different value of parameter $x$ are considered: $7 \mathrm{~mm}, 21 \mathrm{~mm}$, and $35 \mathrm{~mm}$. The contacts opening velocity is constant $10 \mathrm{~m} / \mathrm{s}$, the rated pressure of working gas is $15 \mathrm{~atm}$.

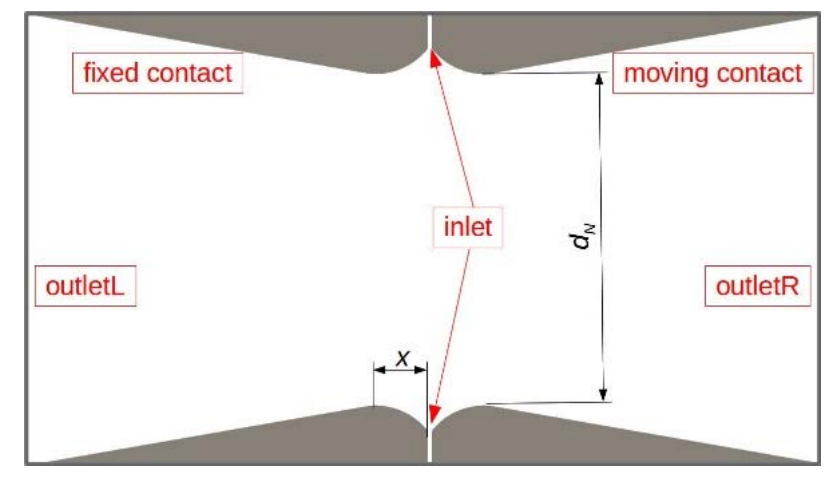

Fig. 3. Model geometry.

The numerical method [19-20] was formed on the basis of free open source package OpenFOAM [21]. To investigate the physical processes in the similar systems of model SCB it is often neglected a movement of working parts for calculation optimisation. However, in this task, for completed analysis of the gas flow arrangement into the switching gap it is necessary to consider interactions between the gas flow and movable elements. It can be reached by the dynamic mesh approach.

To describe the transonic compressible gas flow the Navier-Stokes equations are solved in collaboration with the mass and energy conservation laws. The system of equations is shorted by an equation of state for ideal gases. The numerical method is realised in the OpenFOAM solver rhoCentralDyMFoam with support for mesh-motion and topology changes [22]. The solver was developed for computation of density-based compressible flow, based on central-upwind schemes of Kurganov and Tadmor [23] and finite volume method.

For this task, it was built the irregular mesh. As a tool for the computational grid constructing, the generator 
Table. 1. Boundary conditions.

\begin{tabular}{|c|c|c|c|c|}
\hline & inlet & Outlet & movingWall & fixedWall \\
\hline pressure, $\mathrm{Pa}$ & $\begin{array}{c}\text { totalPressure } \\
15 \mathrm{e} 5\end{array}$ & $\begin{array}{c}\text { waveTransmissive } \\
1 \mathrm{e} 5\end{array}$ & zeroGradient & zeroGradient \\
\hline $\begin{array}{l}\text { temperature, } \\
\mathrm{K}\end{array}$ & $\begin{array}{l}\text { totalTemperature } \\
300\end{array}$ & zeroGradient & zeroGradient & zeroGradient \\
\hline velocity, m/s & $\begin{array}{l}\text { pressureInletOutletVelocity } \\
\qquad\left(\begin{array}{lll}0 & 0 & 0\end{array}\right)\end{array}$ & zeroGradient & movingWallVelocity & noSlip \\
\hline pointMotionU & slip & $\begin{array}{l}\text { uniformFixedValue } \\
0\end{array}$ & $\begin{array}{l}\text { uniformFixedValue } \\
10\end{array}$ & $\begin{array}{c}\text { uniformFixedValue } \\
0\end{array}$ \\
\hline
\end{tabular}

blockMeshDict was chosen. Near computational domain of the contacts opening, it is required to provide a sufficiently small cell size to ensure sufficient accuracy of the calculation and to avoid the cells degeneration with mesh motion. In the computations, a mesh with 318000 cells was used.

The simulation is run in two-dimensional problem statement and in continuous medium approximation. The task is solved with the boundary conditions definition (table 1). A parallel processing algorithm is used in the calculation. To decompose the computational grid, the module decomposeParDict is applied for dividing into subtasks, according to the number of using processors.

The contacts opening is simulated by mesh morphing in the domain between nozzles. For tasks with moving mesh, the boundary conditions must be set with specific keywords to provide the feedback (pointMotionU in table 1). The surface velocity $10 \mathrm{~m} / \mathrm{s}$ is supplied as boundary condition for movingWall to simulate the contact opening. The uniform value of zero only applies to the initialization. The fundamental equations for the compressible flow propagation with mesh morphing are described in [24]. The mesh movement realises by the OpenFOAM module dynamicMeshDict. A Laplace`s equation for the motion velocity rebuilds the mesh around movingWall by use of a program module dynamicMeshDict (object velocityComponentLaplacian with “directional” type of diffusivity):

$$
\boldsymbol{\nabla} \cdot\left(\gamma \nabla \mathbf{U}_{\mathrm{m}}\right)=0,
$$

where $\mathbf{U}_{\mathbf{m}}$ is a velocity vector, $\gamma$ is a diffusion coefficient.

Dry air is taken as arc quenching medium. The fast process is considered adiabatic. Physical properties for the medium are set in the special file thermophysicalProperties.

\section{Results and Discussion}

Results of the numerical simulation for the gas-dynamics parameters are fixed in the process of the contacts opening. The complexity of the pressure, mass and velocity interchanges has the major contribution to the arcing energy release under the synchronous commutation. The knowledge of the cold-gas phenomena provides a good basis for better comprehension of the arc-gas interactions. Time of the gas flow stabilisation is necessary to evaluate lower time limit for the active arc-gas energy interchanges beginning.

Figure 4 presents the calculated time evolution of the critical mass flow $Q_{c r}=\rho U S_{c r}$ at the axis point of the nozzle critical sectors for fixed (a) and moving (b) contacts, where $\rho$ is a density, $U$ is velocity, $S_{c r}$ is a cross sectional area of the nozzle throat. The plots are graphically demonstrated the gas flow stabilisation in 0.8-0.9 ms after the contacts opening start for all types of nozzle arrangement with $x=7 \mathrm{~mm}$ (blue curve), $21 \mathrm{~mm}$ (green curve), $35 \mathrm{~mm}$ (red curve). This provides the effective interactions between cold gas flow and plasma residual column at the most probable position for arc quenching (at the beginning part of a divergent nozzle).

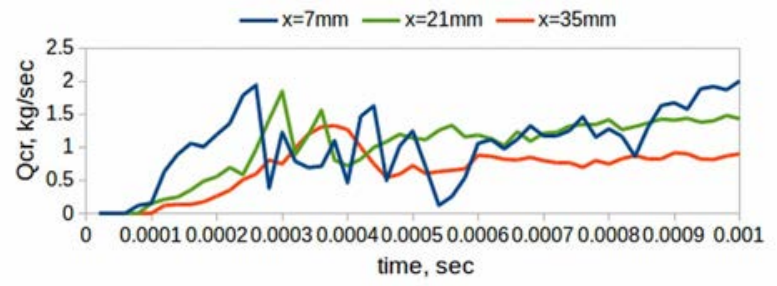

a)

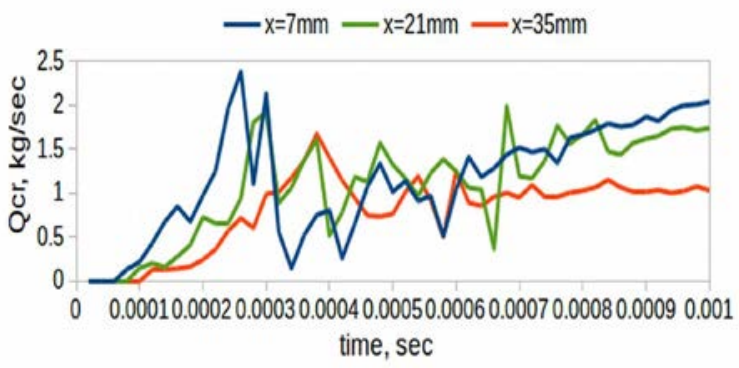

b)

Fig. 4. Mass flow at the nozzle critical sectors of a) fixed contact, b) moving contact.

The curves in fig. 5 show the normalized pressure ratio $p / p_{0}$ in convergent nozzle for various times from the contacts opening start $t=0.7 \mathrm{~ms}$ (dotted line), $t=1.5 \mathrm{~ms}$ (full line), where $p$ is a pressure along the geometry axis, $p_{0}$ is the inlet rated pressure. By this time, a length between contacts corresponds to $7 \mathrm{~mm}$ and $15 \mathrm{~mm}$ for $0.7 \mathrm{~ms}$ and $1.5 \mathrm{~ms}$ accordingly. The zero on the OX-axis 
means a point of the contacts opening start. With a steady flow rate, the mass flow fluctuations are defined by the density and pressure differential. The pressure increasing is known to enhance the interruption ability in accordance of basic relationship in switchgear literature (see introduction).

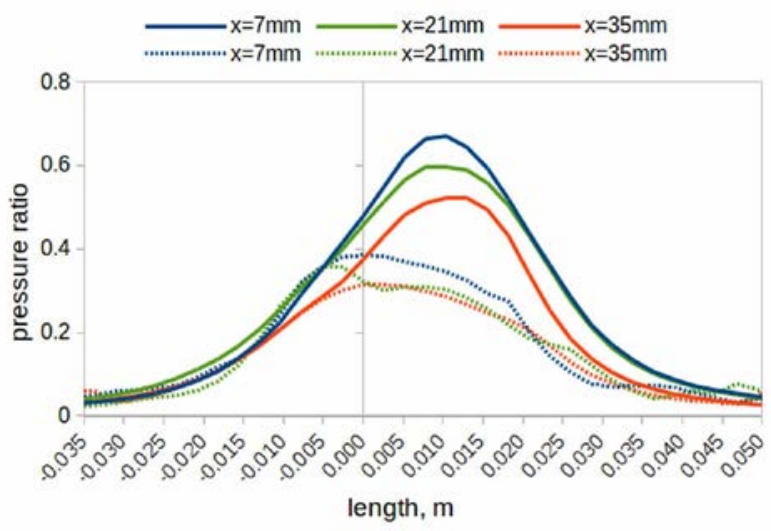

Fig. 5. Pressure ratio along axis in convergent nozzle of different types for various times from the contacts opening: $t=0.7 \mathrm{~ms}$ (dotted line), $t=1.5 \mathrm{~ms}$ (full line).

The experimental results in [7] showed the successful synchronous commutation in model SCB at $1.5 \mathrm{~ms}$ after the contacts opening. Figure 6 demonstrates a comparative analysis of the normalized mass flow $Q^{*}=\rho U /(\rho U)_{c r}$ along the model axis for different types of nozzle geometry at the time $t=1.5 \mathrm{mc}$. The contacts opening length is $15 \mathrm{~mm}$. The critical mass flow $Q_{c r}$ is higher for the investigated model with the nozzle arrangement $x=7 \mathrm{~mm}$ (see fig. 4). But, in spite of the high absolute value of the mass flow, the parameter of normalized mass flow $Q^{*}$ for this nozzle changes insignificantly by comparison with others (see fig. 6). That could reduce the arc-gas energy exchange and activate a formation of the stagnation points in the switching gap. The stagnation zone is characterized by the high temperature, minimum mass discharged, high pressure and reduces recovery processes in the region between electrical contacts.

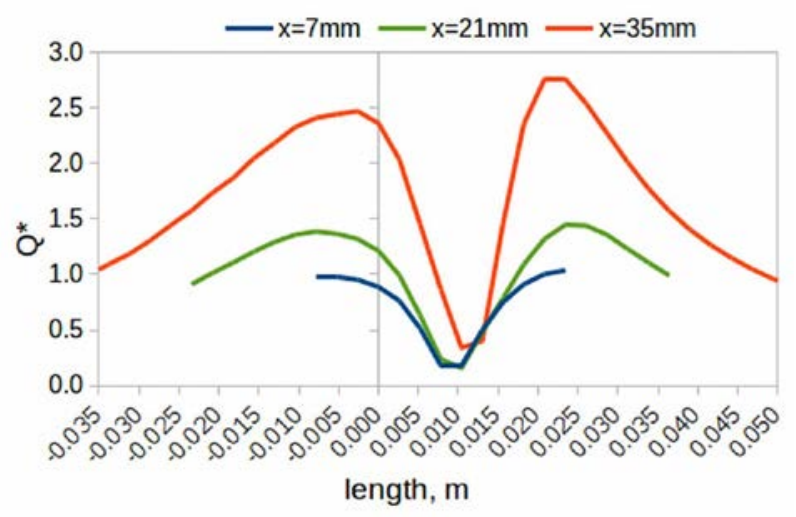

Fig. 6. Normalized $Q^{*}$ in the switching gap along axis for different nozzle arrangement at $t=1.5 \mathrm{~ms}$.

Figure 7 presents colour pressure distribution in the switching gap at $t=1.5 \mathrm{~ms}$ for different types of the nozzle geometry $x=7 \mathrm{~mm}$ (fig. $7 \mathrm{a}$ ), $x=21 \mathrm{~mm}$ (fig.7 b) and $x=35 \mathrm{~mm}$ (fig. 7c). The white dashed lines define a region between the critical sectors of the nozzle.

The stagnation zone influence on the arc quenching process under the synchronous commutation depends upon the convergent nozzle shape and size. Taking into attention normalized ratio $Q^{*}$ (fig. 6), the pressure magnitude in the stagnation points for the model with $x=7 \mathrm{~mm}$ (fig.7a) is higher, than in the other arrangements. Thermal-dielectric recovery starts at the zones of critical nozzle throats, but stagnation points in the upstream region reduces recovery processes. The stagnation region for the geometry with $x=7 \mathrm{~mm}$ (fig.7a) is situated in close vicinity to the nozzle critical sectors and the divergent nozzle (supersonic part). Therefore, a probability of electrical breakdown after current zero increases for this nozzle arrangement of the model SCB.

\section{Conclusions}

Analysis of the effective arc-gas interactions has been carried out in the double-flow air blast interrupter under the synchronous commutation by the numerical method with mesh moving to increase interruption ability of the SCB. The gas flow parameters are investigated for the

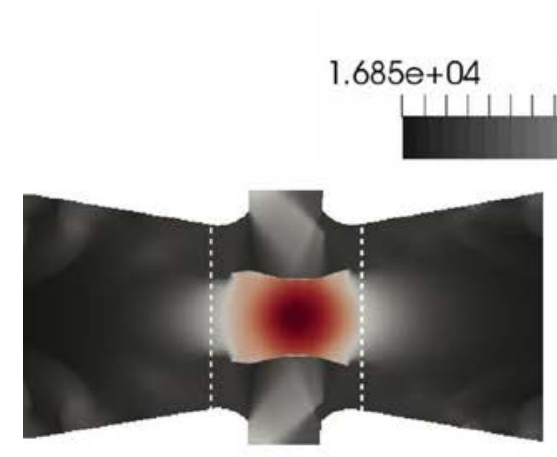

a)

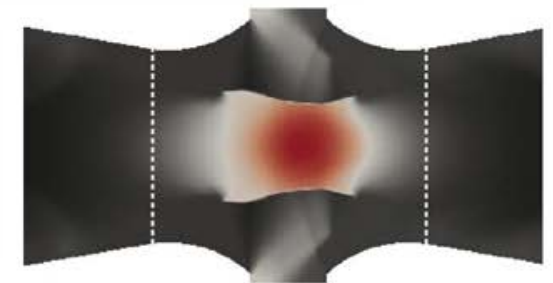

b)

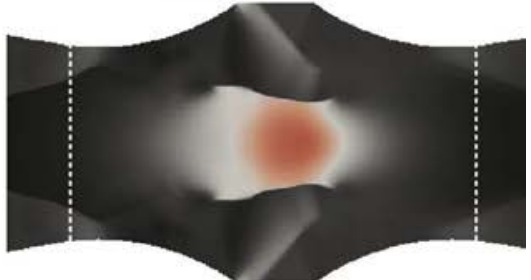

c)

Fig. 7. Analysis of stagnation zone by pressure distribution for different kind of the nozzle geometry at $t=1.5 \mathrm{~ms}(x=7 \mathrm{~mm}$ (a), $x=21 \mathrm{~mm}(\mathrm{~b}), x=35 \mathrm{~mm}(\mathrm{c}))$. 
different types of nozzle arrangements during the contacts opening.

The active gas-arc energy exchange is shown to be began in $t=0.8-0.9 \mathrm{~ms}$ after the contacts opening. Therefore, the lower time limit for the synchronous commutation before current zero is defined.

The stagnation points influent on the arc extinguishing process. The influence is defined the site of arc quenching (either into the nozzle throat or periphery) and depends on the convergent nozzle shape and size (subsonic part). With reducing of the convergent nozzle geometry, the probability of the thermal-dielectric breakdown is enhanced.

\section{Acknowledgements}

The results of the work were obtained using computational resources of Peter the Great SaintPetersburg Polytechnic University Supercomputing Centre (www.spbstu.ru).

\section{References}

1. Current Zero Club. www.currentzeroclub.org

2. Y. Shklyarskiy, A. Shklyarskiy, E. Zamyatin, Tsvetnye Met. 84-91 (2019).

3. H. Hama, S. Okabe, R. Buhler, D. Gautschi, A. Girodet, M. Hering, D. Imamovic, K. Juhre, Y. Kieffel, J. Kindersberger, W. Koltunowicz, J. Lopes-Roldan, S. Neuhold, C. Neumann, R. Pietsch, U. Riechert, T. Rokunohe, U. Schichler, and T. Yasuoka, Dry air, N2, CO2 and N2/SF6 mixtures for gas-insulated systems. Electra, 71 (2018, Aug).

4. Kyoto Protocol to the United Nations Framework Convention on Climate Change (1998) http://unfccc.int/kyoto_protocol/items/2830.php

5. M. Seeger, T. Votteler, S. Pancheshnyi, J. Carstensen, A. Garyfallos, and M. Schwinne, Plasma Physics and Technology 6(1), 39 (2019)

6. I. Vrangov, Arc quenching investigation in $A C$ breakers with synchronous commutation, Thesis for the degree of licentiate of engineering. Leningrad, LPU (1971)

7. T. Morita, M. Iwashita, and Y. Nitta. IEEE Transactions on Power Apparatus and Systems, PAS97(3), 940-949, (1978)

8. C. Cereda, C. Gemme, and C. Reuber, 15th Int. Conf. on Electr. Distr, 73 (1999).

9. R. Thomas. Controlled switching of high voltage SF6 circuit breakers for fault interruption, Thesis for the degree of licentiate of engineering (2004) http://webfiles.portal.chalmers.se/et/Lic/ThomasRichard Lic.pdf.

10. O. Bolshunova, A. Korzhev, and A. Kamyshyan, IOP Conf. Ser. Mater. Sci. Eng. 327, 052007 (2018)

11. A. Briggs, and L. King, IEE Conf. Dev in Design and Performance 52 (1979)

12. A. Briggs, Proceedings of the 7th International Conference on Gas Discharges and their Applications, 28 (1982)
13. G. Frind, and J. Rich, IEEE Transactions on Power Apparatus and Systems, PAS 93(5), 1675 (1974)

14. N. Kurakina, V. Frolov, and E. Tonkonogov, Plasma Physics and Technology, 6(1), 43 (2019)

15. S. Averyanova, and E. Tonkonogov, Plasma Physics and Technology 6(2), 140 (2019)

16. A. Budin, M. Pinchuk., V. Kuznetsov, V. Leontev, and N. Kurakina, Instruments and Experimental Techniques, 60 n.6, 837-842 (2017)

17. E. Tonkonogov, and E. Ugriumov, 14th Symp. Phys. Switching Arc, 149 (2001)

18. G. Alexandrov, A. Afanasev, V. Borisov, G. Kaplan, V. Kuznetsov, V. Lunin, M. Moiseev, E. Tonkonogov, Yu. Filippov, and M. Yarmarkin, High voltage electrical apparatus, $2^{\text {nd }}$ ed. (St. Petersburg: SPbGTU, 2000)

19. N. Kurakina, M. Pinchuk, A. Budin, A. Smirnovsky, and V. Frolov, St. Petersburg polytechnic university journal of engineering science and technology, 24(2), 69 (2018)

20. N. Kurakina, M. Pinchuk, A. Budin, and A. Smirnovsky, Journal of Physics: Conference Series, 1135(1), 012094 (2018)

21. OpenFOAM.The OpenFOAM Foundation, https://openfoam.org/

22. Official OpenFOAM Repository. OpenFOAM. https:/github.com/OpenFOAM/.../ rhoCentralFoam/ rhoCentralDyMFoam

23. A. Kurganov, and E. Tadmor, Journal of Computational Physics, 160 n.1, 241 (2000)

24. J. Ferziger Computational Methods for Fluid Dynamics, 3rd ed. (Berlin; Heidelberg; NewYork; Barcelona; Hong Kong; London; Milan; Paris; Tokyo: Springer, 2002) 